\title{
BMJ
}

\section{Routine care of peripheral intravenous catheters versus clinically indicated replacement: randomised controlled trial}

\author{
Joan Webster, nursing director, research; adjunct professor, ${ }^{1,2}$ Samantha Clarke, clinical nurse, ${ }^{1}$ \\ Dana Paterson, research nurse, ${ }^{1}$ Anne Hutton, clinical nurse, ${ }^{1}$ Stacey van Dyk, research nurse, ${ }^{1}$ \\ Catherine Gale, intravenous nurse specialist, ${ }^{1}$ Tracey Hopkins, clinical nurse consultant, intravenous \\ service ${ }^{1}$
}

${ }^{1}$ Centre for Clinical Nursing, Royal Brisbane and Women's Hospital, Herston, Qld 4029, Australia

${ }^{2}$ Research Centre for Clinical and Community Practice Innovation Griffith University, Centre for Clinical Nursing, Royal Brisbane and Women's Hospital

Correspondence to: J Webster joan_webster@health.qld.gov.au

Cite this as: BMJ 2008;337:a339 doi:10.1136/bmi.a339

\section{ABSTRACT}

Objective To compare routine replacement of intravenous peripheral catheters with replacement only when clinically indicated.

Design Randomised controlled trial.

Setting Tertiary hospital in Australia.

Participants 755 medical and surgical patients: 379 allocated to catheter replacement only when clinically indicated and 376 allocated to routine care of catheter (control group).

Main outcome measure A composite measure of catheter failure resulting from phlebitis or infiltration.

Results Catheters were removed because of phlebitis or infiltration from 123 of 376 (33\%) patients in the control group compared with 143 of 379 (38\%) patients in the intervention group; the difference was not significant (relative risk 1.15, 95\% confidence interval 0.95 to 1.40 ). When the analysis was based on failure per 1000 device days (number of failures divided by number of days catheterised, divided by 1000), no difference could be detected between the groups (relative risk $0.98,0.78$ to 1.24). Infusion related costs were higher in the control group (mean \$A41.02; £19.71; €24.80; \$38.55) than intervention group (\$A36.40). The rate of phlebitis in both groups was low ( $4 \%$ in intervention group, $3 \%$ in control group).

Conclusion Replacing peripheral intravenous catheters when clinically indicated has no effect on the incidence of failure, based on a composite measure of phlebitis or infiltration. Larger trials are needed to test this finding using phlebitis alone as a more clinically meaningful outcome.

Registration number Australian New Zealand Clinical Trials Registry ACTRN12605000147684.

\section{INTRODUCTION}

Intravenous catheterisation is the most common invasive procedure among patients admitted to hospital, with about half receiving intravenous therapy during their stay. ${ }^{1}$ The procedure is not without risks. Between 2.3\% and $67 \%$ of patients develop thrombophlebitis; the rate depending on definitions used and populations studied..$^{2-8}$ The more serious complication, infection of the bloodstream, occurs in about $0.1 \%$ of cases. $^{9}$

Many interventions have been developed to reduce the incidence of phlebitis, including new catheter materials, ${ }^{1011}$ innovative methods for securing the catheter, ${ }^{12} 13$ and heparinised catheters. ${ }^{14}$ The most widely practised intervention, however, is routine replacement of the catheter. The Centers for Disease Control and Prevention advocate replacing catheters every 72-96 hours to limit the potential for infection, ${ }^{15}$ but the recommendation is based on scant evidence. ${ }^{16}$ More recent observational studies indicate that catheters may be safely left in place for longer periods. ${ }^{171117}$ Some evidence also suggests that phlebitis is more likely to occur in the two days after catheterisation. ${ }^{1718}$ Consequently, removing a functional catheter and resiting it might expose patients to unnecessary risk.

Despite the ubiquitous use of peripheral intravenous catheters and the almost universal acceptance of the need for routine replacement, the practice has received little rigorous evaluation. We found a total of four randomised controlled trials comparing some form of routine catheter replacement with peripheral venous infusion. Two of these trials were small, carried out in a gastroenterology unit of Scarborough Hospital in the United Kingdom, and enrolled patients only receiving total parenteral nutrition through a peripheral venous catheter. ${ }^{1920}$ Because of the uniqueness of this group, results are not relevant to the current discussion. The third study was carried out in the same unit of the Scarborough Hospital. ${ }^{21}$ It was the first trial to compare outcomes between groups in a general hospital population where the peripheral catheter was either routinely replaced or changed only when clinically indicated. In the control group $(n=26)$, peripheral venous catheters were replaced every 48 hours and in the intervention group removed "if the site became painful, the cannula dislodged or there were signs of peripheral vein thrombosis" $(\mathrm{n}=21)$. Phlebitis occurred in $1(5 \%)$ patient in the control group and $11(42 \%)$ in the intervention group. The study was criticised for its small sample size (sample size and power estimates were not reported) and the involvement of the chief 
investigator in both the randomisation process and the outcome assessment. ${ }^{22}$ Moreover, the incidence of phlebitis in the intervention group was much higher than contemporary rates.

We recognised the need to test current standards using an appropriately powered randomised controlled trial. In March 2004 we began a study using a small sample to compare the safety and efficacy of replacing intravenous catheters after three days with only when clinically indicated. ${ }^{23}$ Challenging the guidelines from the Centers for Disease Control and Prevention made the trial controversial and so we limited our sample to patients in whom the catheter had been inserted by the intravenous therapy team; a group of nurses with extensive collective experience and expertise in the area. Also we excluded patients who were immunocompromised, had a current bloodstream infection, or were unable to sign consent. We randomised participants into two groups (routine care of the catheter or replacing the catheter only when clinically indicated) and used a composite measure of any unplanned reason for removal of the catheter as our primary outcome, including phlebitis, blockage, or infiltration. The secondary outcome was cost. Among the 206 participants studied we found no significant differences between groups on the primary outcome. The duration of peripheral catherisation was similar in both groups (mean 123.3 hours in the intervention group, mean 125.9 hours in the control group) but significantly more re-siting occurred in the control group (166 resites in the intervention group, 216 resites in the control group). Three cases of phlebitis occurred (one in the intervention group, two in the control group).

\section{Potentially eligible participants $(n=1620)$}

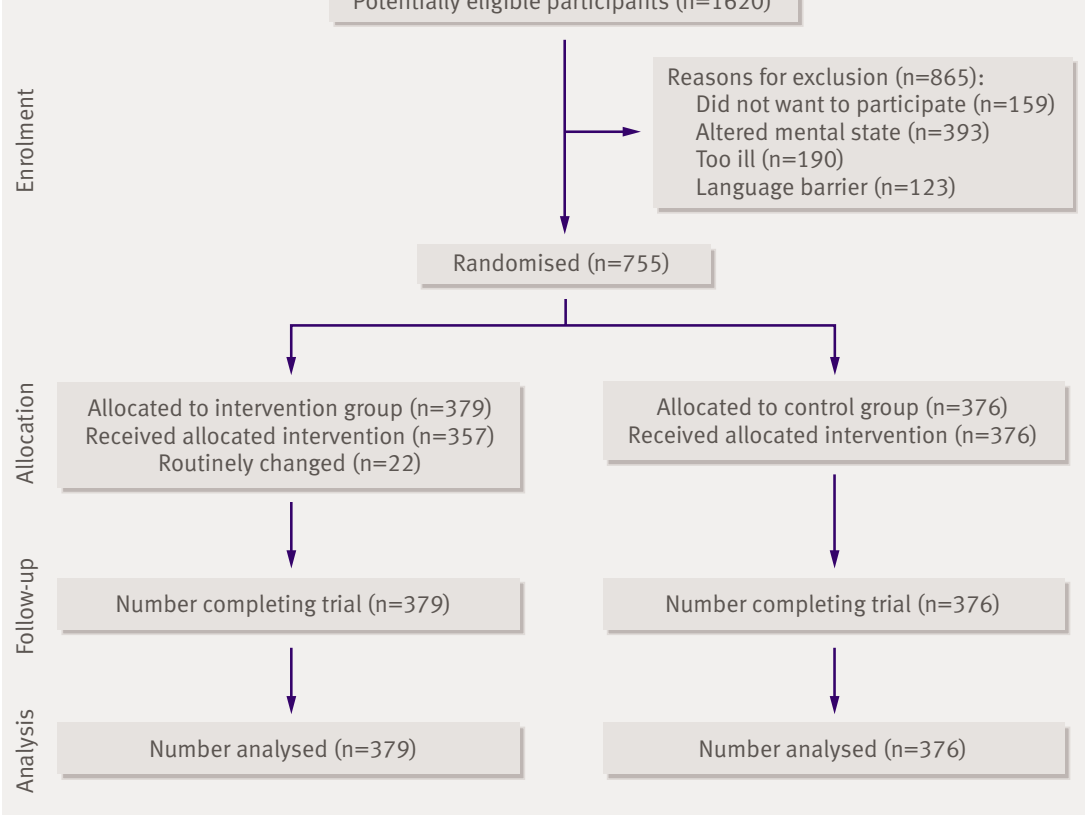

Flow of participants through trial
As a follow-up to this study we carried out a randomised trial to compare routine changes of catheters with clinically indicated changes among a broader group of acute patients admitted to hospital. We used a pragmatic design that excluded few patients and that was carried out under routine hospital procedures. We tested the safety and efficacy of the process on a wider population.

\section{METHODS}

We recruited inpatients from a general tertiary referral teaching hospital (982 beds) with several specialties, including medicine, surgery, orthopaedics, psychiatry, oncology, obstetrics, gynaecology, and trauma services. The hospital is the largest in Queensland, Australia, and provides services to patients throughout the state, northern New South Wales, the Northern Territory, and from neighbouring countries in the south west Pacific. Patients were eligible for inclusion if they were at least 18 years of age, had no current bacteraemia, were not receiving immunosuppressive therapy, and were scheduled or expected to have a peripheral venous catheter for at least four days. As the patient was the unit of measurement for the study we entered patients requiring multiple or consecutive catheters into the study once only. In the earlier trial we had to exclude many, otherwise eligible, participants because an altered mental state prevented them from giving informed consent. For the current trial we sought permission from the hospitals' human research ethics committee for the next of kin to sign for patients who were unable to consent themselves.

Intervention, allocation concealment, and masking We randomised patients either to routine care (control group), with catheters scheduled to be replaced every three days according to hospital policy, or to a group where the peripheral intravenous catheter was not replaced unless there was a clinical indication (clinically indicated group). Irrespective of the assignment, a decision to change a catheter at any time because of clinical indications such as phlebitis, infiltration, or unexplained fever could be implemented. We recorded the reason for replacement of catheters and deviations from the catheter replacement protocol for each group.

Block randomisation was by a computer generated random number list prepared by an investigator with no clinical involvement in the trial. We stratified by admission for an oncology related procedure. After the research nurse had obtained the patient's consent, she telephoned a contact who was independent of the recruitment process for allocation consignment.

Allocation concealment avoided selection bias but clinical staff were subsequently aware of the treatment group. This was necessary to ensure that catheters scheduled for routine replacement were changed and that those in the clinically indicated group were not removed if the catheter was functional and there was no sign of local inflammation or infection. Research staff had no involvement in nominating the reason for 
catheter removal or in diagnosing phlebitis. Staff in the microbiological laboratory were blind to group assignment of catheters submitted for testing.

\section{Procedures}

After consent and randomisation the research nurse collected baseline personal, clinical, and catheter related data, which included the type of infusate and any additives, drugs injected into the intravenous set and their $\mathrm{pH}$ level, type and size of catheter used, and the site of insertion. Choice of catheter type and gauge was at the discretion of the nurse or doctor inserting the catheter. Catheter insertion sites were inspected daily by a nurse from the intravenous therapy team and by ward nurses when intravenous solutions were changed or when drugs were added. Because research staff were not responsible for recording the reason for catheter removal, a specially designed form was developed for completion by the nurse removing the cannula. This was to optimise the standardisation of reporting outcome measures.

Any deviations from the protocol were recorded (if the catheter was replaced at a time other than indicated by the allocated group). We collected data for up to five consecutive catheters for each patient.

\section{Outcome measures}

For the primary outcome we used a composite measure of catheter failure as a result of phlebitis or infiltration. Phlebitis was defined as the presence of two or more of pain, tenderness, warmth, erythema, swelling, or a palpable cord. ${ }^{1-25}$ Infiltration was defined as

Table 1| Baseline personal, clinical, and catheter related details of patients allocated to routine care of intravenous catheter (control group) or to replacement of catheter only when clinically indicated (intervention group). Values are numbers (percentages) of patients unless stated otherwise

\begin{tabular}{|c|c|c|}
\hline Variable & $\begin{array}{l}\text { Intervention group } \\
\quad(n=379)\end{array}$ & Control group $(n=376) \dagger$ \\
\hline Mean (SD) age (years) & $60.1(17.1)$ & $58.8(18.8)$ \\
\hline Male & $248(65)$ & $233(62)$ \\
\hline \multicolumn{3}{|l|}{ Type of admission: } \\
\hline Medical & $238(63)$ & $214(57)$ \\
\hline Surgical & $141(37)$ & $162(43)$ \\
\hline$>1$ comorbidity & $280(74)$ & $270(72)$ \\
\hline Oncology related condition & $55(15)$ & $56(15)$ \\
\hline Current wound infection (including ulcers) & $100(26)$ & $70(19)$ \\
\hline History of phlebitis & $13(3)$ & $4(1)$ \\
\hline Indwelling urinary catheter & $63(17)$ & $79(21)$ \\
\hline Wound drain & $72(19)$ & $43(11)$ \\
\hline Mean (SD) haemoglobin level on admission & $126.4(23.7)$ & $124.5(23.5)$ \\
\hline Skin turger fair or poor & $233(62)$ & $228(61)$ \\
\hline High risk of acquiring nosocomial infection & $36(10)$ & $30(8)$ \\
\hline Poor veins & $127(34)$ & $143(38)$ \\
\hline \multicolumn{3}{|l|}{ Catheter inserted by: } \\
\hline Intravenous team & $502(74)$ & $563(74)$ \\
\hline Medical officer & $66(10)$ & $58(8)$ \\
\hline Other nurse (not intravenous team) & $113(17)$ & $140(18)$ \\
\hline
\end{tabular}

Person inserting catheter was unknown in 14 instances. permeation of intravenous fluid into the interstitial compartment, causing swelling of the tissue around the site of the catheter.

We used several secondary outcomes including infusion related cost, which was calculated in two ways - costs associated with catheters inserted for the intermittent administration of intravenous drugs, and cost associated with catheters inserted for continuous infusion. For patients receiving intermittent drugs (estimated to be $52 \%$ of the population on the basis of data from the earlier trial) we calculated a total cost of \$A16.4. This included 20 minutes nursing per medical time (locating patient, preparation, and insertion), a catheter, a three way tap, a basic dressing pack, gloves, a syringe, transparent adhesive dressing, skin disinfection, and local anaesthetic per insertion. For patients receiving a continuous infusion we calculated a total cost of \$A28.84 per insertion. This included the costs of the initial cathether set plus the cost of replacing associated lines, solutions, and additives, which are discarded when a catheter is replaced (intravenous administration set, 1 litre sodium chloride $0.09 \%$ ). We also included other reasons for catheter failure as secondary outcomes: occlusion or blockage, local infection at the insertion site (defined as erythema with cellulitis at the site or pus at the site), or catheter related bloodstream infection (on the basis of the isolation of a phenotypically identical organism from a catheter segment and a blood culture). ${ }^{11} \mathrm{We}$ also included phlebitis and infiltration as independent secondary outcomes.

\section{Statistical analysis}

We based our sample size on the combined incidence of phlebitis and infiltration in controls from our earlier study $(33.5 \%) .{ }^{23}$ To detect a difference of at least $30 \%$ between groups with a two sided test, $\alpha$ of 0.05 , and $90 \%$ power we determined that we needed about 380 patients in each arm.

Trial data were entered using SPSS version 15 and analysed on an intention to treat basis (all randomised patients were analysed in the assigned groups). A validity check was undertaken on a random sample of $5 \%$ of the data. We compared baseline clinical and personal characteristics to test adequacy of the randomisation process. We calculated the relative risks and 95\% confidence intervals for the proportion of patients with a failed catheter and for the individual factors that made up the composite measure. We also calculated the failure rate for each group per 1000 device days, which is a more meaningful measure for this outcome, by dividing the total number of catheter failures by the total number of days catheterised, multiplied by 1000 . When appropriate, we used a two sided Fisher's exact test to compare discrete data; results are presented as $\mathrm{P}$ values. We used the independent sample $t$ test to compare the differences in the infusion related costs and total catherisation time between the groups. The guidelines for the consolidated standards of reporting trials ${ }^{26}$ were followed from the point of recruitment. 
Table 2 Mean (SD) length of time each catheter remained in place in patients allocated to routine care of intravenous catheter (control group) or to replacement of catheter only when clinically indicated (intervention group)

\begin{tabular}{|c|c|c|c|c|}
\hline Group & No & Mean (SD) & Mean difference $(95 \% \mathrm{Cl})$ & $P$ value* \\
\hline Control & 372 & $66.5(28.1)$ & \multirow{2}{*}{$-24.12(-130.53$ to -17.71$)$} & \multirow{2}{*}{$<0.000$} \\
\hline Intervention & 379 & $90.6(56.5)$ & & \\
\hline Control & 190 & $59.7(30.6)$ & \multirow{2}{*}{$-14.98(-23.94$ to -6.01$)$} & \multirow{2}{*}{0.001} \\
\hline Intervention & 161 & $74.7(53.3)$ & & \\
\hline Control & 102 & $55.7(28.1)$ & \multirow{2}{*}{$-17.49(-30.24$ to -4.74$)$} & \multirow{2}{*}{0.007} \\
\hline Intervention & 82 & $57.3(73.2)$ & & \\
\hline Control & 52 & $60.4(34.7)$ & \multirow{2}{*}{$-26.74(-50.22$ to -3.26$)$} & \multirow{2}{*}{0.026} \\
\hline Intervention & 34 & $87.1(73.7)$ & & \\
\hline Control & 33 & $57.6(33.8)$ & \multirow{2}{*}{$-3.67(-25.81$ to 18.47$)$} & \multirow{2}{*}{0.741} \\
\hline Intervention & 23 & $61.2(49.0)$ & & \\
\hline
\end{tabular}

*Two sided $t$ test.

\section{RESULTS}

Between February 2006 and June 2007, 755 of 1620 potentially eligible participants $(46.6 \%)$ were included in the trial. Almost one quarter of potentially eligible patients were excluded because of an altered mental state. These were generally patients who were confused or had some form of dementia or delirium. Reasons for exclusion are shown in the figure.

Of those recruited, 376 were randomised to routine care of catheters (control group) and 379 to replacement of catheters only when clinically indicated (intervention group). Twenty two participants $(6 \%)$ in the intervention group had their catheter changed routinely rather than in response to a clinical indication. One hundred and twenty five participants (33\%) in the control group had a catheter in place for more than 72 hours, reflecting the variation in routine care. Follow-up from medical records was possible for all participants.

Baseline personal, clinical, and catheter related characteristics were similar between the groups for most risk factors (table 1). The exceptions were a

Table 3 | Outcomes for patients allocated to routine care of intravenous catheter (control group) or to replacement of catheter only when clinically indicated (intervention group). Values are numbers (percentages) of patients unless stated otherwise

\begin{tabular}{|c|c|c|c|}
\hline Outcomes & $\begin{array}{l}\text { Intervention group } \\
\qquad(\mathrm{n}=379)\end{array}$ & Control group $(n=376)$ & Relative risk $(95 \% \mathrm{Cl})$ \\
\hline \multicolumn{4}{|l|}{ Primary: } \\
\hline $\begin{array}{l}\text { Catheter failure per } \\
\text { person }\end{array}$ & $143(38)$ & $123(33)$ & 1.15 (0.95 to 1.40$)$ \\
\hline $\begin{array}{l}\text { Catheter failure per } 1000 \\
\text { device days }\end{array}$ & 59.8 & 60.9 & \\
\hline \multicolumn{4}{|l|}{ Secondary: } \\
\hline $\begin{array}{l}\text { Mean (SD) intravenous } \\
\text { cost per catheter }\end{array}$ & $41.05(26.6)$ & $46.22(28.7)$ & $-5.16^{*}(-9.12$ to -1.21$)$ \\
\hline Phlebitis & $16(4)$ & $12(3)$ & 1.32 (0.63 to 2.76$)$ \\
\hline Infiltration & $135(36)$ & $120(32)$ & 1.12 (0.91 to 1.36$)$ \\
\hline Blockage & $30(8)$ & $20(5)$ & 1.49 (0.86 to 2.57$)$ \\
\hline Local infection & $2(1)$ & 0 & $4.96(0.24$ to 102.98$)$ \\
\hline $\begin{array}{l}\text { Suspected bloodstream } \\
\text { infection }\end{array}$ & $1(0.3)$ & $1(0.3)$ & 0.99 (0.06 to 15.80$)$ \\
\hline
\end{tabular}

*Mean difference. history of phlebitis and presence of a wound infection or infected ulcer, with higher rates in the intervention group.

Each catheter was in place for a greater mean length of time in the intervention group than in the control group (table 2). As a result more catheters were placed in the control group $(n=749)$ than in the intervention group $(n=679)$, despite the average number of intravenous therapy days being less in the control group $(t=$ $-2.77, \mathrm{P}=0.007$; two sided $t$ test): 6.3 (SD 5.1) days in the intervention group and 5.4 (SD 3.8) days in the control group. The total number of days catheterised were 2020 in the control group and 2393 in the intervention group.

\section{Effect of intervention}

Table 3 shows rates for the primary and secondary outcomes. Overall, $123(33 \%)$ participants in the control group and $143(38 \%)$ in the intervention group had catheters removed as a result of phlebitis or infiltration; the difference was not significant (relative risk 1.15, 95\% confidence interval 0.95 to 1.40). When the analysis was based on catheter failure per 1000 device hours, no difference could be detected between the groups. Infusion related costs were higher in the control group per episode of care than in the intervention group (mean \$A41.02 v mean \$A36.40). Both groups had a low rate of phlebitis (4\% in intervention group, 3\% in control group). Infiltration was the most common reason for failure $(36 \%$ in intervention group, 32\% in control group). A total of $196(26 \%)$ catheters in control participants were replaced after three days, according to hospital policy, despite functioning well.

\section{DISCUSSION}

The routine replacement of peripheral intravenous catheters has no effect on the incidence of catheter failure, on the basis of a composite measure of phlebitis or infiltration. The result replicates findings from our earlier study, which used narrower inclusion criteria but a broader definition of failure. ${ }^{23}$ These two studies have increased our confidence in changing intravenous lines according to clinical signs and symptoms, rather than using predetermined time frames. ${ }^{15}$ Changing our policy would bring the practice in adults in line with recommendations from the Centers for Disease Control and Prevention for changing peripheral intravenous lines in children - that is, to replace catheters only when clinically indicated. Paradoxically, in our hospital peripheral intravenous lines are not routinely changed in premature infants or certain other patients in whom achieving and maintaining venous access is difficult. Such populations are arguably at higher risk of developing catheter related complications than general medical and surgical patients in whom routine changes are mandated.

Our overall combined rate for phlebitis and infiltration was $35 \%$, similar to other reports. ${ }^{27} 28$ The phlebitis rate in both groups was on the low side of ranges reported in recent studies, ${ }^{2930}$ despite our population 


\section{WHAT IS ALREADY KNOWN ON THIS TOPIC}

Peripheral intravenous catheterisation is the most common invasive procedure among inpatients

Changing catheters every three days to prevent infection is standard procedure but the practice has not been rigorously tested

\section{WHAT THIS STUDY ADDS}

Catheters may be safely left in place for longer than 72 hours if no contraindications are present

When catheters are replaced only when clinically indicated $25 \%$ of infusion related costs are saved

being elderly and unwell, with almost $75 \%$ having at least one comorbidity. Reported rates depend on definitions used, and although we applied a standard definition, interpretation of signs and symptoms could still be affected by subjectivity or omission of reporting. For example, it has been argued that infiltration (easy to diagnose) may result from unrecognised phlebitic changes to the vein wall (hard to diagnose) leading to under-reporting of phlebitis. ${ }^{27} \mathrm{It}$ is perhaps more useful to use the composite measure of infiltration or phlebitis as it avoids any potential for misdiagnosis.

Despite allocation to the control or intervention group, participants showed little difference in dwell times. Two factors contribute to this. Firstly, it is not possible to modify all routinely scheduled changes precisely 72 hours after insertion - for example, some catheters may be left to the next morning rather than waking a patient during the night. Secondly, for various reasons many of the catheters in the intervention group failed before 72 hours-although catheters in the intervention group remained in place longer than those in the control group, the average dwell time was within the 72-96 hours recommended by the Centers for Disease Control and Prevention. This confirms that all catheters fail eventually but that many remain functional for prolonged periods. We found that about $3 \%$ remained trouble free for over seven days and some for as long as two weeks. Because of this we believe that routinely changing catheters may be an unnecessary and painful intervention for patients, and costly for the organisation.

Potential cost savings of about $25 \%$ for infusion related costs could be made if our policy was to be changed in line with recent evidence. Cost estimates used in our study were conservative, based on a simple intravenous event. Others have suggested that about $2.5 \%$ of total drug costs are wasted when preparations are destroyed. ${ }^{31}$ On the basis of recent data from England this could translate to a cost saving of about $£ 61 \mathrm{~m}$ per annum. ${ }^{32}$

The study was not sufficiently powered to show differences in our secondary clinical outcomes. Despite this, non-significant results favoured the control group for lower rates of phlebitis, blockage, and local infection. Numbers were small for these outcomes and confidence intervals wide but with a larger enrolment this risk could reach statistical significance.
A large, multicentre trial is needed to confirm our results, using phlebitis or bloodstream infection as the primary outcome. Several serious adverse events were reported during the trial. None of these were related to trial procedures.

\section{Strengths and limitations}

The major strengths of the study were the processes used to eliminate selection bias, to ensure allocation concealment, and to ensure that the study was adequately powered to detect differences in our primary outcome. We also included a broad range of participants and did not impose any caveats on how or by whom catheters should be inserted. This was to match normal clinical practice and to ensure that results could be extrapolated to other populations of complex inpatients. We were able to enrol $47 \%$ of potentially eligible patients compared with about $25 \%$ in the earlier trial. Most of those we were unable to recruit were either too frail or their mental state prevented them from providing informed consent. From the point of randomisation, no losses to follow-up occurred.

The study would have been strengthened if monitoring of outcomes had been more stringent. As it was, we extracted most of the outcome data from medical records. A more standardised approach would have been preferable, using staff trained in the process and data collected in real time. Another potential limitation was that outcome assessment was done by people who were not blinded to group allocation. Although catheters were removed by ward staff or intravenous service staff, part of their normal practice is to record reasons for removal in the patient's medical record. To falsify such an observation because of group allocation would be unlikely, so we believe our results have not been compromised. Finally, the study was not powered to study differences in the secondary outcomes. Phlebitis alone would have been a more clinically important end point but we were limited by restrictions on funding. For the same reason we did not culture catheter tips. This may have provided additional information to inform practice and future studies.

\section{Conclusion}

Replacing peripheral intravenous catheters only when clinically indicated does not reduce the incidence of catheter failure, on the basis of a composite measure of phlebitis or infiltration. Larger trials are needed to test this finding using phlebitis alone as a more clinically meaningful outcome.

Contributors: JW conceived the idea, based on earlier work, secured funding, wrote the protocol, supervised the preparation and conduct of the trial, analysed the data, and wrote the paper. SE developed databases and assisted in the development of study materials and in data management. DP collected data for primary outcomes and developed a system for categorising the $\mathrm{pH}$ of drugs. $\mathrm{AH}$ was responsible for assessing suitability and supervising recruitment of acute admissions. SvD collected data for primary outcomes, helped in the development of study materials, and collated data. CD liaised with the intravenous service, hospital staff, and participants, and assisted in data management. CG contributed to the 
protocol and assisted with expert advice throughout the trial. All authors contributed to and approved the final draft. JW is guarantor.

Funding: Queensland Nursing Council research grant (RAN:0513) and the RBWH Research Foundation.

Competing interests: None declared.

Ethical approval: Royal Brisbane and Women's Hospital human research ethics committee (No 2005/93)

Provenance and peer review: Not commissioned; externally peer reviewed.

1 Bregenzer T, Conen D, Sakmann P, Widmer AF. Is routine replacement of peripheral intravenous catheters necessary? Arch Intern Med 1998;158:151-6.

2 Tripepi-Bova KA, Woods KD, Loach MC. A comparison of transparent polyurethane and dry gauze dressings for peripheral IV catheter sites: rates of phlebitis, infiltration, and dislodgment by patients. Am J Crit Care 1997;6:377-81.

3 Chee S, Tan W. Reducing infusion phlebitis in Singapore hospitals using extended life end-line filters. / Infus Nurs 2002;25:95-104.

4 Tager IB, Ginsberg MB, Ellis SE, Walsh NE, Dupont I, Simchen E, et al. An epidemiologic study of the risks associated with peripheral intravenous catheters. Am J Epidemiol 1983;118:839-51.

5 Martinez JA, Fernandez P, Rodriguez E, Sobrino J, Torres M, Nubiola A, et al. Intravenous cannulae: complications arising from their use and analysis of their predisposing factors. Med Clin (Barc)

1994;103:89-93.

6 Lipsky BA, Peugeot RL, Boyko EJ, Kent DL. A prospective study of Staphylococcus aureus nasal colonization and intravenous therapy related phlebitis. Arch Intern Med 1992;152:2109-12.

7 White SA. Peripheral intravenous therapy-related phlebitis rates in an adult population. J Intraven Nurs 2001;24:19-24.

8 Shimandle RB, Johnson D, Baker M, Stotland N, Karrison T, Arnow PM Safety of peripheral intravenous catheters in children. Infect Control Hosp Epidemiol 1999;20:736-40.

9 Maki DG, Kluger DM, Crnich CJ. The risk of bloodstream infection in adults with different intravascular devices: a systematic review of 200 published prospective studies. Mayo Clin Proc 2006;81:1159-71.

10 Gaukroger PB, Roberts JG, Manners TA. Infusion thrombophlebitis: a prospective comparison of 645 Vialon and Teflon cannulae in anaesthetic and postoperative use. Anaesth Intensive Care 1988;16:265-71.

11 Cornely OA, Bethe U, Pauls R, Waldschmidt D. Peripheral Teflon catheters: factors determining incidence of phlebitis and duration of cannulation. Infect Control Hosp Epidemiol 2002;23:249-53.

12 Callaghan S, Copnell B, Johnston L. Comparison of two methods of peripheral intravenous cannula securement in the pediatric setting. J Infus Nurs 2002;25:256-64.

13 Wood D. A comparative study of two securement techniques for shor peripheral intravenous catheters. J Intraven Nurs 1997;20:280-5.

14 Shah PS, Ng E, Sinha AK. Heparin for prolonging peripheral intravenous catheter use in neonates. Cochrane Database Syst Rev 2005:CD002774.
15 O’Grady NP, Alexander M, Dellinger EP, Gerberding JL, Heard SO, Maki DG, et al. Guidelines for the prevention of intravascular catheterrelated infections. Infect Control Hosp Epidemiol 2002;23:759-69.

16 Lai KK. Safety of prolonging peripheral cannula and iv tubing use from 72 hours to 96 hours. Am J Infect Control 1998;26:66-70.

17 Homer LD, Holmes KR. Risks associated with 72- and 96-hour peripheral intravenous catheter dwell times. J Intraven Nurs 1998;21:301-5.

18 Catney MR, Hillis S, Wakefield B, Simpson L, Domino L, Keller S, et al. Relationship between peripheral intravenous catheter dwell time and the development of phlebitis and infiltration. J Infus Nurs 2001;24:332-41.

19 Kerin MJ, Pickford IR, Jaeger H, Couse NF, Mitchell CJ, Macfie J. A prospective and randomised study comparing the incidence of infusion phlebitis during continuous and cyclic peripheral parenteral nutrition. Clin Nutr 1991;10:315-9.

20 May J, Murchan P, MacFie J, Sedman P, Donat R, Palmer D, et al. Prospective study of the aetiology of infusion phlebitis and line failure during peripheral parenteral nutrition. Br J Surg 1996;83:1091-4.

21 Barker P, Anderson AD, MacFie J. Randomised clinical trial of re-siting of intravenous cannulae. Ann R Coll Surg Engl 2004;86:281-3.

22 Idvall E, Gunningberg L. Evidence for elective replacement of peripheral intravenous catheter to prevent thrombophlebitis: a systematic review. I Adv Nurs 2006;55:715-22.

23 Webster J, Lloyd S, Hopkins T, Osborne S, Yaxley M. Developing a research base for intravenous peripheral cannula re-sites (DRIP trial). A randomised controlled trial of hospital in-patients. Int I Nurs Stud 2007;44:664-71.

24 Maki DG, Ringer M. Risk factors for infusion-related phlebitis with small peripheral venous catheters. A randomized controlled trial. Ann Intern Med 1991;114:845-54.

25 Monreal M, Oller B, Rodriguez N, Vega J, Torres T, Valero P, et al. Infusion phlebitis in post-operative patients: when and why. Haemostasis 1999;29:247-54.

26 Moher D, Schulz KF, Altman DG. The CONSORT statement: revised recommendations for improving the quality of reports of parallelgroup randomized trials. Ann Intern Med 2001;134:657-62.

27 Wright A, Hecker J. Infusion failure caused by phlebitis and extravasation. Clin Pharm 1991;10:630-4.

28 Garland JS, Dunne WM Jr, Havens P, Hintermeyer M, Bozzette MA, Wincek J, et al. Peripheral intravenous catheter complications in critically ill children: a prospective study. Pediatrics 1992;89:1145-50.

29 Malach T, Jerassy Z, Rudensky B, Schlesinger Y, Broide E, Olsha O, et al. Prospective surveillance of phlebitis associated with peripheral intravenous catheters. Am J Infect Control 2006;34:308-12.

30 Nassaji-Zavareh M, Ghorbani R. Peripheral intravenous catheterrelated phlebitis and related risk factors. Singapore Med ) 2007;48:733-6

31 Uchida KM, Iteen SA, Wong NT. Identifying costs of intravenous solution wastage. Pharmacoeconomics 1994;6:240-8

32 Anon. Hospital drug costs rise. Hospital Pharmacist 2007;14:322.

Accepted: 7 May 2008 